\title{
Ophthalmic artery aneurysm treated by surgical clipping and balloon-assisted temporary carotid occlusion
}

\begin{abstract}
Amir R. Dehdashti, M.D.
Department of Neurosurgery, Northshore University Hospital, Hofstra Northshore LIJ School of Medicine, Manhasset, New York

A 29-year-old woman is diagnosed with a large broad-base right ophthalmic artery aneurysm. Despite an intact visual field, she complained of mild right visual blurriness. Between endovascular and surgical treatments, surgery was favored due to her young age. It was decided to perform the surgery with simultaneous endovascular temporary carotid balloon occlusion. A right pterional craniotomy and intradural anterior clinoidectomy were performed, the balloon was positioned in the petrous carotid, and the distal dural ring was opened exposing the proximal neck. Under temporary proximal carotid balloon occlusion and distal carotid clip occlusion, the aneurysm was fully dissected and clipped. Intraoperative angiogram confirmed complete aneurysm occlusion and patency of the ophthalmic artery. The patient's neurological exam remained unchanged.
\end{abstract}

The video can be found here: http://youtu.be/BIQKTI9bDqA.

KEYWORDS aneurysm; microsurgery; ophthalmic artery; clinoidectomy; balloon occlusion; video 